W. Braeutigam: Ueber das Tiliadin.

Gefunden :

Berechnet für

Hg. $38,04 \%$ $\mathrm{C}_{9} \mathrm{H}_{11} \mathrm{NO}_{3} \cdot \mathrm{CH}_{8} \mathrm{Cl} \cdot \mathrm{HgCl}_{2}$

$\mathrm{C}_{9} \mathrm{H}_{13} \mathrm{NO}_{4} \cdot \mathrm{CH}_{8} \mathrm{Cl} \cdot \mathrm{Hg} \mathrm{Cl}_{\varepsilon}$ :

Cl. 20,09 , $39,89 \%$ $38,42 \%$ 21,19 , 20,46 ,

Die für $\mathrm{Hg}$ und $\mathrm{Cl}$ gefundenen Werte würden für die für die Formel $\mathrm{C}_{9} \mathrm{H}_{11} \mathrm{NO}_{3} \mathrm{CH}_{3} \mathrm{ClHg} \mathrm{Cl}_{2}$ berechneten zu niedrig sein und wäre nur noch die Annahme möglich, dass die Verbindung noch ein Mol. $\mathrm{H}_{2} \mathrm{O}$ enthielt, welches beim Trocknen über Schwefelsäure nicht abgegeben würde.

Das Damasceninchlorid liefert zum Unterschiede von dem Methylchloridadditionsprodukt mit $\mathrm{Hg} \mathrm{Cl}_{2}$ keine Doppelverbindung.

Schneider berichtet zwar, dass Sublimat mit Damasceninsalzen sofort eine weisse Fällung giebt; indessen erhielt ich dieselbe nicht.

Vorstehende Daten übergebe ich zunächst der Oeffentlichkeit, ohne damit die weitere Untersuchung des Damascenins abzuschliessen. Eine Reihe von bereits begonnenen Versuchen konnte wegen Mangel an Material noch nicht beendigt werden und behalte ich mir daher deren Veröffentlichung in einer späteren Mitteilung vor.

\title{
Ueber das Tiliadin, einen Bestandteil der Lindenrinde.
}

Von Dr. Walter Braeutigam.

(Eingegangen den 1. VIII. 1900.)

Eine vorläufige Mitteilung über einen cholesterinartigen Körper in der Rinde der Linde hatte ich bereits im Jahre 1898 in der "Pharm. Ztg." No. 105 veröffentlicht. Damals hatte ich die Beobachtung gemacht, dass der Rückstand des Aetherauszuges der frischen Lindenrinde, eine bräunliche klebrige Masse, mit $90 \%$ kaltem Alkohol behandelt, sich nur zum Teil löste, während der unlösliche Teil, in Aether gelöst, nach dessen Verdunsten eine gelbe klebrige Substanz hinterliess, in welcher sich, unter dem Mikroskop betrachtet, Krystalle zeigten.

Um nun die Krystalle von der klebrigen Beimengung, welche eine harzähnliche Masse war, zu trennen, wurde der Aetherrückstand mit 5\% Kalilauge gekocht, das Unlösliche auf einem Filter gesammelt und so lange mit warmem Wasser ausgewaschen, bis das Ablaufende eine alkalische Reaktion nicht mehr zeigte.

Den geringen Filterrückstand behandelte ich, nachdem er getrockuet war, mit Aether. Darin löste er sich. Hierauf liess ich die 
Aethersolution auf einem Uhrschälchen verdunsten und erhielt eine kleine Menge atlasglänzender Krystalle, die nur geringe Verunreinigungen erkennen liessen.

Das günstige Resultat dieser Vorprüfung veranlasste mich, grössere Mengen frischer Lindenrinde in Arbeit zu nehmen. Die Rinde wurde zunächst mit Aether $(0,720)$ erschöpft und der so erhaltene Auszug filtriert. Nach dem Verdunsten des Aethers wusch ich den Rückstand so lange mit kaltem $90 \%$ Alkohol aus, bis derselbe sich nicht mehr färbte.

Die Untersuchung der alkoholischen Lösung ergab, dass diese, ausser einer klebrigen Masse, auch noch geringe Mengen von Vanillin enthielt, aber keine der früher erwähnten Krystalle.

Das im Alkohol Unlösliche war ebenfalls von klebriger Beschaffenheit und wurde zunächst mit $5 \%$ Kalilauge behufs Verseifung der harzähnlichen Masse gekocht. Danach wurde das durch den Verseifungsprozess Nichtgelöste auf einem Filter gesammelt und hierauf mit heissem Wasser ausgewaschen. Alsdann trocknete ich den Rückstand urd löste ihn in Aether auf. Eine Probe dieser Solution, auf dem Uhrschälchen zur Verdunstung gebracht, liess unter dem Mikroskope atlasglänzende Kryställchen erkennen. In dieser waren noch geringe Verunreinigungen zu beobachten, die hauptsächlich aus beigemengter Seife bestanden. Um diese nun zu entfernen, verdunstete ich den Aether, nahm den Rückstand mit heissem, absolutem Alkohol auf und versetzte diese Lösung mit einer wässerigen Solution von Chlorcalcium, um die vorhandene geringe Menge von Seife in Kalkseife zu verwandeln.

Hierauf verdampfte ich das Ganze auf dem Wasserbade bis zur Trockne und extrabierte dann mit Aetber. Die Aetherlösung liess nach dem Verdunsten die Krystalle fast rein erscheinen, und durch wiederholtes Umkrystallisieren aus heissem, absolutem Alkohol oder beissem Essigäther konnten diese frei von fremden Beimengungen erhalten werden.

Das Auswaschen des Aetherrückstandes mit Alkohol hat verschiedene Vorteile. Zunächst geht die Verseifung schneller vor sich und das Entfärben der Krystalle vermittelst Tierkoble fällt weg; ausserdem hat man nicht nötig, behufs Entfernung des Vanillins die ätherische Lösung mit einer wässerigen Solution von Natriumbisulfit auszuschütteln, da dessen geringe Menge in den Aetherauszug ïbergeht.

Bei meinen weiteren Versuchen über eine bequemere Darstellungsweise der genannten Krystalle hatte ich beobachtet, dass, wenn man mehrere Aetherauszüge in ein und demselben Kolben destilliert, sich aus den erhaltenen konzentrierten Lösungen betr. Krystalle an der Wandung und am Boden des Destillier-Kolbens ansetzen. Will man 
die ausgeschiedenen Krystalle, welche sich unter dem Mikroskope als Plättchen von verschiedener Grösse, Form und Stärke zeigten, ohne Verseifung gewinnen, so hat man nur dafür zu sorgen, dass genügend Aether in dem Destillierkolben zurückbleibt, damit die klebrige Masse in Lösung erhalten wird. Hierauf stellt man den Kolben zum weiteren Absetzen der Krystalle an einen nicht zu kühlen Ort, da sich sonst in der Kälte oft harzartige Substanzen abscheiden, welche dann das Reinigen der Krystalle erschweren. Nachdem dies geschehen ist, giesst man die überstehende Flüssigkeit, worin sich die etwa vorhandenen Krystalle von Vanillin gelöst befinden, von den ausgeschiedenen Krystallen $a b$ und reinigt sie durch Umschütteln mit wenig kaltem Essigäther von der anhängenden klebrigen Masse. Durch wiederholtes Umkrystallisieren aus heissem Essigäther oder Alkohol abs. erhält man die reinen Krystalle, welche aus Plättchen von verschiedener Grösse und Stärke bestehen und den Cholesterinkrỹ tallen sehr ähneln. Sollen die in der Aetherlösung zurückgeblieben . srystalle gewonnen werden, so verfährt man wie früher angegeben.

Die Ausbeute der Krystalle, welche äusserst leicht sind, ist sehr gering; denn ein Kilo Lindenrinde giebt ungefähr 0,3 bis 0,5 Gramm. Die so gewonnenen Krystalle zeigten sich unter dem Mikroskop als Plättchen, jedoch konnte ich auch Krystallnadeln erkennen.

Bei meinen weiteren Untersuchungen hatte ich beobachtet, dass die nadelförmigen Krystalle oft ganz verschwanden, wäbrend die Plättchenformen immer vorhanden waren.

Ich fand, dass die nadelförmigen Gebilde besonders stark auftraten, wenn ich die Rinde von der Westseite des Baumes entnommen hatte, welche zum grössten Teile mit Grünalgen bedeckt war. Entfernte ich den grünen Belag sorgfältig mittelst Bürsten vor der Bearbeitung der Rinde, so bemerkte ich nur ganz vereinzelte NadelKrystalle.

Diese Beobachtung veranlasste mich, den grünen Algenanflug zu sammeln und zu untersuchen.

Zunächst liess dessen vorgenommene mikroskopische Prüfung ihn als Pleurococcus vulgaris erkennen.

Wurde dieser mit Aether ausgezogen, so schieden sich beim Verdunsten die oben beobachteten nadelförmigen Krystalle aus. Dieselben Krystallformen wurden erhalten, wenn man an Stelle des Aethers Alkohol abs. oder Essigäther verwendete. Diese Untersuchung hatte mir gezeigt, dass der grüne Algenanflug, bestehend aus Pleurococcus vulgaris, jene beobachteten Krystallnadeln lieferte, über welche ich in einer späteren Arbeit berichten werde.

Da diese Krystallnadeln sich bedentend leichter in denen hier 
in Frage kommenden Lösungsmitteln auflösten als die Krystallplättchen, so war eine Trennung beider leicht möglich.

Die Krystalle, über welche diese Arbeit handelt, waren geruchund geschmacklos und bestanden in der Hauptsache aus farblosen, glänzenden Plättchen, deren Gestalt und Grösse abhängig war von der Art und der Konzentration des Lösungsmittels, sowie von der Temperatur, bei welcher sie auskrystallisierten. Aus einer heissen konz. Solution von absolutem Alkohol, 90\%igem Spiritus, Aether und Essigäther scheiden sie sich als ein weisses Pulver aus, das, unter dem Mikroskope betrachtet, aus im Grunde rechteckigen dünnen Plättchen bestand, während sie aus einer konz. kalten alkoholischen Lösung, welche man der Selbstverdunstung überliess, sich als makroskopische Krystallplättchen absonderten. In Xylol sind sie leicht löslich und scheiden sich aus einer konz. heissen Solntion desselben als lange Plättchen aus, welche oft zu Kreuzen, bezw. Sternen verbunden waren.

Liess ich $a_{c}$ ren eine kaltgesättigte Xylollösung mit diesen Krystallen ohne Anwendung von Wärme verdunsten, so erhielt ich lange Plättchen von fast $2 \mathrm{~cm}$ Grösse.

In kaltem, wie in kochendem Wasser blieben sie ungelöst, ebenso in Alkalien. Unlöslich zeigten sie sich ferner in Salz- und Salpetersäure, und es konnte, nachdem sie auf dem Wasserbade mit ihnen zur Trockne verdampft waren, eine äusserliche Veränderung der Krystalle nicht wahrgenommen werden. In kalter Schwefelsäure lösten sich die Krystalle mit roter Farbe, welche beim Erwärmen in Schwarz überging. Löslich waren die Krystalle in Aether $(0,720$ Spez. Gew. $=0,225 \%$, Essigäther, Alkohol abs., Spiritus $(90 \%=0,084 \%)$, Chloroform $(6 \%)$, Essigsäureanhydrid, Phenol, Benzol, Eisessig, Schwefelkohlenstoff, Pyridin, Ligroin und Benzin.

Die Lösungen reagierten neutral, und Polarisationsversuche mit Chloroformlösungen zeigten eine Rechtsdrehung. Ferner liessen sich die Krystalle beim vorsichtigen Erhitzen unter gewöhnlichem Druck unzersetzt verflüchtigen.

Das Verhalten dieses Körpers zu Alkalien und Säuren, sowie zu den verschiedenen Lösungsmitteln und seine Darstellungsweise veranlassten mich $\mathrm{zu}$ der Vermutung, dass er eine cholesterinartige Masse sei.

Um nun näheren Aufschluss darüber zu erlangen, wurden mit den aufgefundenen Krystallen Cholesterinreaktionen vorgenommen.

Zunächst versuchte ich die von $\mathrm{Hesse}^{1}$ ) angegebene Prüfung, welche auf dem Verhalten von cholesterinartigen Körpern zu Schwefel-

1) Ann. der Chemie Bd. 211, 283. Hesse, Salkowsky, Zeitschr. für analyt. Chemie Bd. $26,572$. 
säure und Chloroform beruht. Einige Centigramme von den fraglichen Krystallen wurden in $2 \mathrm{~cm}$ Chloroform gelöst, hierauf $2 \mathrm{ccm}$ Schwefelsäure hinzugefügt und die Mischung dann umgeschüttelt. Bei Gegenwart von Cholesterin und bei Phytosterin soll die Chloroformlösung sich blutrot, dann kirschrot bis purpurn färben, während die Schwefelsänre eine starke grüne Fluorescenz zeigt. In meinem Falle färbte sich die Chloroformlösung rot und nahm später eine rotviolette Färbung an, während sich die Schwetelsäure braunrot färbte und schwach fluorescierte.

Eine weitere Prüfungsmethode ist die von Liebermann ${ }^{1}$ ). Dieser fand, dass, wenn man reine konzentrierte Schwefelsäure in eine kalt gehaltene, gesättigte Lösung von Cholesterin in Essigsäureanhydrid träufelt, sich die Lösung vorübergehend blau färbt.

Bei der Prüfung der fraglichen Substanz entstand zunächst auf Zusatz einiger Tropfen von konzentrierter reiner Schwefelsäure eine rötliche Färbung der unteren Schicht, die auf weiteres Zutropfen an Umfang gewann, während die obere Schicht gelblich erschien, und beim leichten Schwenken des Reagierglases sich gleichfalls rötlich färbte, welche Färbung bald in Rotgelb uberging. Andere Prüfungen, welche vorgenommen wurden, waren die von $S$ chiff ${ }^{2}$ ). Dieser fand, dass, wenn eine kleine Menge Cholesterin mit einem Tropfen Salpetersäure gelinde erwärmt wurde, nach diesem ein gelber Fleck zurückbleibt, der, noch warm mit $\mathrm{HN}_{3}$ übergossen, rot wird (Unterschied des Cholesterins von Harnsäure).

Bei meinen Versuchen mit den betr. Krystallen konnte ich eine Rotfärbung nicht wahrnehmen, wohl aber eine gelblichrote.

Der Schmelzpunkt dieser Krystalle wurde zu $228-229^{\circ} \mathrm{C}$. ermittelt. Die bis jetzt ausgeführten Schmelzpunktbestimmungen der bekannten cholesterinartigen Körper schwanken zwischen 125 und $160^{\circ} \mathrm{C}$. und nur das Lupeol $^{8}$ ) weist einen Schmelzpunkt von $204^{\circ} \mathrm{C}$. auf.

In neuerer Zeit hat Thoms ${ }^{4}$ ) in einer vorläufigen Mitteilung über Cerin, wofür er die empirische Formel $\mathrm{C}_{82} \mathrm{H}_{54} \mathrm{O}_{2}$ aufgestellt hat, und welches er zu den Phytosterinen zählt, den Schmelzpunkt dieser Substanz zu $249^{\circ} \mathrm{C}$. ermittelt. Demnach würde das Cerin den höchsten Schmelzpunkt von den bis jetzt bekannten cholesterinartigen Körpern besitzen.

Auf Grund dieser Beobachtungen hatte ich in meiner vorläufigen Mitteilung diesen krystallinischen Körper, welchen ich, zum Unter-

1) Ber. der chem. Ges. Bd. 18, 1804.

2) Ann. der Chemie Bd. 115, 313 .

8) E. Schmidt, Pharm. Chemie 1896, Bd. 2, 643.

H. Thoms, Ueber die chemischen Bestandteile des Korkes. Pharm. Centralh. 1898, No. 39. 
schiede von Tiliacin ${ }^{1}$ ), einem Glykosid aus den Lindenblättern, Tiliadin genannt habe, in die Reihe der Phytosterine (Pflanzencholesterine) gestellt. Wie weit ich hierzu berechtigt war, wird sich aus den hier angeführten Versuchsreihen ergeben.

Die Untersuchung des Tiliadins auf Stickstoff ergab mit Natronkalk, sowie nach dem Verfahren von Lassaigne und Dumas ein negatives Resultat. Die Elementaranalysen ergaben folgende Daten:

\begin{tabular}{|c|c|c|}
\hline \multicolumn{2}{|c|}{ Substanz ergaben $\mathrm{CO}_{2}$} & $\mathrm{H}_{2} \mathrm{O}$ \\
\hline I. $0,0973 \mathrm{~g}$ & 0,2851 & 0,0908 \\
\hline II. 0,126 & 0 , & \\
\hline III. 0,10 & 0,3142 & 01 \\
\hline IV. 0,1300 & 0,3815 & \\
\hline
\end{tabular}

Hieraus berechnen sich folgende Prozente an $\mathrm{C}$ und $\mathrm{H}$ :

I. II. III. IV.

C. $79,9 \quad 80,34 \quad 80,16 \quad 80,04$

H. $10,38 \quad 10,35 \quad 10,43 \quad 10,27$.

woraus sich die Formel $\mathrm{C}_{21} \mathrm{H}_{83} \mathrm{O}_{2}$ ergiebt.

Die ausgeführten Molekulargewichtsbestimmungen bestätigen die Richtigkeit dieser Formel; denn der Formelausdruck $\mathrm{C}_{21} \mathrm{H}_{32} \mathrm{O}_{2}$ verlangt ein Molekulargewicht von 316 und gefunden wurde 297.

Nach der Auffindung der empirischen Formel stellte ich nun Versuche an, um zu einem Aufschluss über die Konstitution dieses Körpers zu gelangen. Zunächst behandelte ich die betr. Krystalle mit Salzsäure.

$\mathrm{Zu}$ diesem $\mathrm{Zwecke}$ erhitzte ich je $1 \mathrm{~g}$ Tiliadin mit konzentrierter Salzsäure 5 Stunden lang auf $150-160^{\circ} \mathrm{C}$. Beim Oeffnen der Einschmelzrohre war nur ein ganz geringer Druck zu konstatieren. Die so behandelten Krystalle liessen äusserlich keine Veränderung erkennen, auch die vorgenommenen Krystallisationsversuche zeigten gleichfalls keine Abänderung; auch der Schmelzpunkt war derselbe geblieben. Bei den Einschlussversuchen hatten demnach die physikalischen Eigenschaften der Tiliadin-Krystalle eine Veränderung nicht erfahren; doch wurde vorsichtshalber noch eine Prüfung der Krystalle auf einen event. Chlorgehalt vorgenommen.

Das Resultat war jedoch ein negatives. (Glähen mit gebranntem Marmor.) Demnach hat also die Salzsäure weder eine physikalische noch eine chemische Veränderung der Krystalle hervorgerufen.

Hieran schlossen sich die Versuche mit den Halogenen. Von diesen konnten die Chlor- und Bromverbindungen dargestellt werden. Eine Verbindung des Tiliadins mit Jod konnte dagegen, trotz verschieden vorgenommener Versuche, nicht erhalten werden.

1) Vergl. E. Schmidt 1896, Bd. 2, 1583. 